\title{
A CASE STUDY FOR SUMMARY WRITING WITH A GRAPHIC ORGANIZER IN WEB-BASED INVESTIGATIVE LEARNING
}

\author{
Oriko Harada and Akihiro Kashihara \\ Graduate School of Informatics and Engineering, The University of Electro-Communications \\ 1-5-1, Chofugaoka, Chofu, 182-8585, Tokyo, Japan
}

\begin{abstract}
In Web-based investigative learning (WBIL), learners construct knowledge on a question by navigating and collecting information from Web resources. Previous works have designed a learning model as well as a cognitive tool to support learners to conduct deep and wide investigation instead of building superficial knowledge. On the cognitive tool, learners can create a learning scenario representing the navigation path between Web resources, save keywords extracted from Web resources, create a table of contents (TOC) to linearize their knowledge, and build text structure for the summary of a paper to report their respective investigations. In Harada and Kashihara (2020), we proposed a method for summary writing in WBIL, where learners use graphic organizers (GOs) to structure the summary. The current paper will report on the between-subjects case study conducted to evaluate this method. Results showed that while there was little difference between the experimental and contrast group, learners in the experimental group considered the method effective for organizing and visualizing their knowledge.
\end{abstract}

\section{KEYWORDS}

Web-Based Investigative Learning, Summary Writing, Graphic Organizer, Learner-Created Learning Scenario

\section{INTRODUCTION}

With the prevalence of personal computers and mobile devices, gathering information online has become an everyday activity. The Web has become the main information resource especially for students, who have been reported to use information online to complete most of their assignments (Julien and Barker, 2009). Information seeking on the Web consists of multiple processes, such as selecting suitable resources and navigating between pages, and various research has been conducted to provide support. For example, Nishihara et al (2006) aimed to support learners in selecting resources according to their knowledge of the topic. To this end, they developed an application which extracts keywords from Web resources and provides learners with a difficulty of each resource and the order of reading resources to understand a certain topic. Aramaki et al (2016) aimed at supporting elementary school students to internalize what they read from resources by providing a tablet application where students can save images from resources and a note attached to each image. Our aim, on the other hand, is to deepen and widen the learners' investigation on the Web. To do so, we have defined a learning model where learners seek information with not only a single query but multiple search queries.

"Web-based investigative learning (WBIL for short)" is defined as a form of learning where learners use search engines to investigate any question with Web resources to construct knowledge. In WBIL, learners are provided with a learning model and a cognitive tool where they can create a learning scenario visualized as a tree with each search query as its node and the progress of one search query to another as links between the nodes. WBIL targets beginners in searching information online, but basic knowledge of using computers and reading skills to comprehend online articles are presupposed. In practice, university students would be the main targets of WBIL, and our past experiments have been conducted on undergraduate and graduate students (Kashihara and Akiyama, 2016; Morishita et al, 2020; Harada and Kashihara, 2020). The learning model and iLSB has then progressed to encompass not only investigation online but also reflecting, organizing and presenting knowledge constructed from resources. The current work focuses on the last learning process, where learners are expected to write a paper reporting the investigation. 
While information on iLSB, such as keywords extracted from resources and learning scenarios, are useful for the learners themselves in investigating or reflecting upon information, they cannot be used by other learners to understand another learners' investigation. For the learners to communicate their learning outcomes to peers or instructors, they must convert the information stored in iLSB into a paper. On the other hand, the paper includes everything a learner investigated and contains a substantial amount of information for the readers of the paper. Therefore, a summary for the paper is necessary as an advance organizer for learners to effectively communicate what they investigated. As there have been no established method for learners of WBIL to write a summary, which itself is not a simple task, our previous work (Harada and Kashihara, 2020) proposed a method to support summary writing in WBIL. This method uses graphic organizers which represent the text structure of a summary for learners to organize information. The current paper focuses on the evaluation of our method and will report on a case study conducted with 16 participants.

\section{SUPPORTING SUMMARY WRITING FOR WBIL}

In this chapter, we will briefly discuss how summary writing for Web-based investigative learning (WBIL) is supported with the learning support system iLSB (interactive Learning Scenario Builder) (Akiyama and Kashihara, 2016) and graphic oranizers (Harada and Kashihara, 2020).

\subsection{Web-based Investigative Learning (WBIL)}

WBIL aims to support learners using Web resources to collect information (Akiyama and Kashihara, 2016). A learning model and a learning support system iLSB (interactive Learning Scenario Builder) was designed. In the model, In the model, learners begin their investigation with a question, which is called the "initial question." Learners construct knowledge for the initial question in the "Knowledge construction process" by expanding the initial question into sub-questions and creating a learning scenario on the iLSB. Next, in the "Knowledge linearization process", learners order and hierarchize their knowledge by creating a table of contents (TOC) from the learning scenario. The goal of creating the TOC is for the learners to write a paper for the learner to report on their findings. The paper consists of a summary and body. Our work focuses on supporting learners in writing the summary. We will explain the framework in the next section.

\subsection{Framework}

Our method uses graphic organizers (GOs) to support learners in organizing various pieces of information stored in the iLSB (Harada and Kashihara, 2020). Graphic organizers represent the text structure of the summary, which is categorized into the following five types (Roehling et al, 2017): description, sequence and order, cause and effect, compare and contrast, and problem and solution. Figure 1 shows GOs for each text structure. Given the table of contents (TOC), iLSB generates a list of GOs based on the relationships between nodes in the TOC. Learners then supplement the information in GOs by adding "node annotations" and "link annotations" on the iLSB. Node annotations are information added to the nodes of a GO and selected by learners from the keywords collected in the knowledge construction process. Link annotations are information for links between nodes in a GO, where learners describe relationships between nodes in their own words.

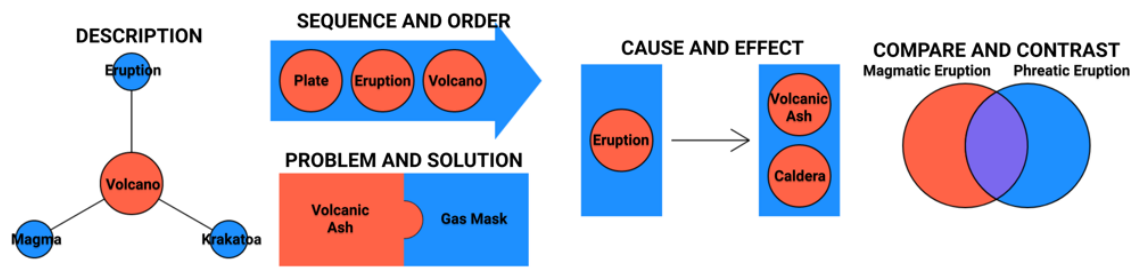

Figure 1. GOs and corresponding text structure 


\subsection{Interactive Learning Scenario Builder (iLSB)}

Interactive Learning Scenario Builder (iLSB) is a Firefox add-on we developed as a cognitive scaffolding (tool) for iLSB (Akiyama and Kashihara, 2016). In the knowledge construction process, learners first enter keyword representing the initial question into a search engine. This keyword is called the "q-keyword". For example, the keyword for the initial question "What is "tax'?" would be "tax". Next, they select resources to gather information from the list of Web pages given by the search engine. For each resource, the learners read the contents and save relevant keywords in the "keyword repository" of iLSB. Once learners feel they have gathered enough information for the initial question, they select the next question to investigate, or the "sub-question". To do so, learners select a new keyword for further investigation among the repository keywords and add them as child nodes in the "question tree", which is a tree structure representing the learning scenario. For each sub-question, learners repeat the above process to enter the q-keyword in the search engine, navigate Web resources, extract keywords, and discover sub-questions. Once learners feel there is no need for expanding questions into sub-questions, the knowledge construction process is completed.

In the knowledge linearization process, learners aim to write a written report on their findings from the knowledge construction process. For this, learners first create a TOC for their paper using iLSB (Morishita et al, 2020). Then, learners structure the summary with the screens shown below. First, learners view the TOC they constructed (Figure 2), from which iLSB generates a list of GOs (Figure 3). Learners then add node and link annotations as shown in Figure 4 and Figure 5. Finally, iLSB displays a list of GOs with annotations.

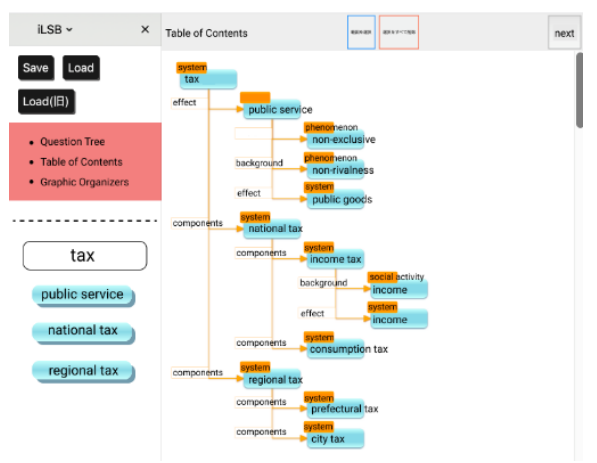

Figure 2. An example of TOC

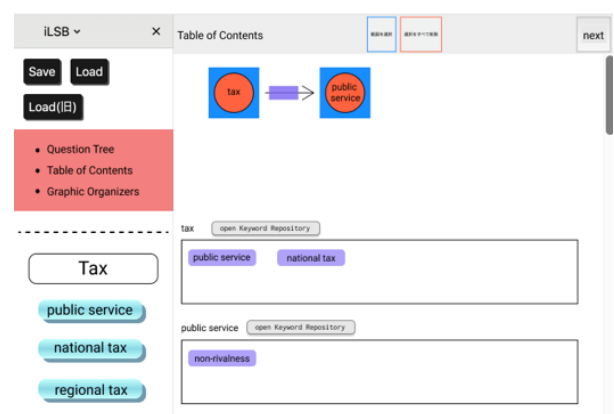

Figure 4. Adding node annotations

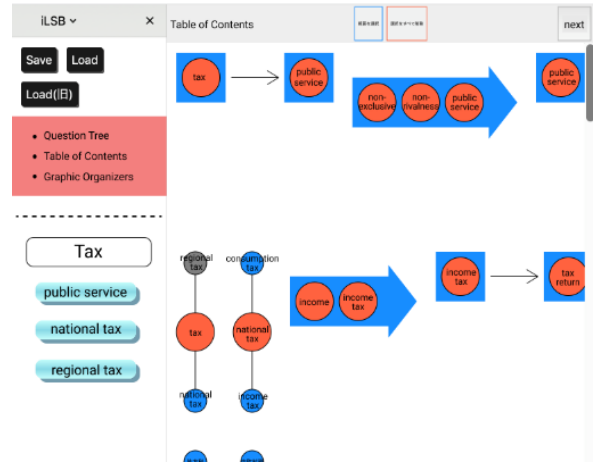

Figure 3. List of GOs

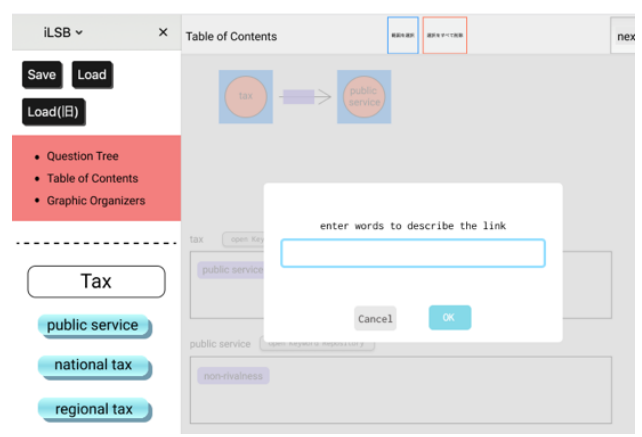

Figure 5. Adding link annotations

\section{CASE STUDY}

To assess the effectiveness of our method in helping learners come up with a better written report more easily, we conducted a case study. We evaluated the proposed method on 1) exhaustiveness of the summary with respect to TOC, and 2) cognitive load of writing the summary with two learner groups. 


\subsection{Methods}

Sixteen undergraduate and graduate students at the University of Electro-Communications, Japan, participated as subjects of this experiment. As the university focuses on informatics and engineering education, all subjects are assumed to have sufficient computer literacy and therefore meet the criteria for WBIL targets. The case study was conducted under a between-subjects design. As the case study was planned to take 210 minutes at the maximum, within-subjects design was considered too time-consuming and exhausting for the subjects. Moreover, potential learning effects would confound any differences found in the study. As such, subjects were equally divided into either the experimental group (8 subjects) and the contrast group (8 subjects).

The subjects first used iLSB for investigating the initial q-keyword "Tax", then created TOC for the paper to report on their investigation also using iLSB, and finally wrote their summaries. In the summary writing, the experimental group could use graphic organizers to build text structure from their TOC and to view the text structure while writing the summary. On the other hand, the contrast group was required to write their summaries using only the TOC and did not create graphic organizers.

Data were collected according to the two evaluation perspectives mentioned above: exhaustiveness of the summary and cognitive load of summary writing. For the former, we analyzed the summaries for its exhaustiveness and letters per q-keyword. We also asked for a subjective assessment of how exhaustive subjects were able to write their summaries in questionnaire \#1, answered both by the experimental and contrast group. For the latter, the summaries were analyzed for writing duration per letter and the number of letters. Questionnaire \#1 asked the subjective difficulty of writing a summary, and questionnaire \#2, answered only by the experimental group, asked the ease of use for iLSB in creating GOs as well as their general impressions on our method.

\subsection{Results}

\subsubsection{Analysis of Summary}

To analyze the summary text, we collected data for 1) exhaustiveness of summary, 2) length of summary, and 3 ) seconds spent for writing the summary. The results for the contrast and experimental group are shown in Table 1.

First, 1) the exhaustiveness of summary was calculated as the ratio of q-keywords in summary to q-keywords in TOC. The contrast group on average covered 91\% (SD: 0.12) of the TOC q-keywords in their summary, and the experimental group average was $96 \%$ (SD: 0.06). For 2) the length of summary, we collected the total number of letters of the summary as well as the number of letters per q-keyword. For the former, the contrast group average was 332 letters (SD: 99.71) and the experimental group average was 558 letters (SD: 343.74). For the latter, the contrast group average was 29.43 letters/q-keyword (SD: 13.96) and the experimental group average was 42.97 letters/keyword (SD: 12.08). Finally, for 3) seconds spent for writing the summary, the contrast group on average took 4.05 seconds/letter (SD: 1.77 ) and the experimental group average was 2.97 seconds/letter (SD: 0.99).

While there were trends between the contrast group and experimental group where the experimental group tended to cover more of the q-keywords in TOC, take less time to write their summaries, and write more for each q-keyword, there was no statistically significant difference between the two groups.

Table 1. Results of summary analysis

\begin{tabular}{lllll}
\hline Data & Contrast & & \multicolumn{2}{c}{ Experimental group } \\
\cline { 2 - 5 } & Average & SD & Average & \\
\hline Q-keywords in summary/ & $91 \%$ & 0.12 & $96 \%$ & \\
q-keywords in TOC & & & & \\
\# of letters & 332 letters & 99.71 & 558 letters & 343.74 \\
\# of letters/q-keyword & 29.43 letters/keyword & 13.96 & 42.97 letters/keyword & 12.08 \\
seconds/letter & 4.05 s/letter & 1.77 & 2.97 s/letter & 0.99 \\
\hline
\end{tabular}




\subsubsection{Reviewer Assessment}

In the reviewer assessment, we asked two reviewers to first read each summary and then to draw TOC which can be assumed that the summary was written from. TOC for each subject by the two reviewers were then compared to the original TOC created by the subject, and the Tree Edit Distance (TED) (Zhang and Shasha, 1989) between TOCs were calculated. This assessment aimed to measure how well the summaries reflected the structure and contents of TOC.

The correlation between the two reviewers were calculated to be 0.71 . As such, we have used the average of the two reviewer's TED to be the distance between reviewer's TOC and subject's TOC. The average TED for the contrast group was 8.88 (SD: 2.98) and 6.38 (SD: 3.31) for the experimental group. While a trend was seen for the experimental group to have shorter TED than the contrast group, there was no statistical significance.

As it was perceived that some subjects in the contrast group possessed high writing skills having shorter TEDs than the experimental group average, we attempted to evaluate the difference between the contrast and experimental groups by separating the former into sub-groups with low skill level and high skill level and comparing the experimental group with the contrast group's low skill level subgroup. This subgroup consisted of three subjects who had longer TEDs than the contrast group average. The results showed the contrast subgroup's average to be 11.67 (SD: 2.83). A t-test between the experimental group and the contrast subgroup showed the two group's difference to be statistically significant at the 0.05 level $(\mathrm{p}=0.049)$.

\subsubsection{Questionnaire \#1}

Questionnaire \#1 was asked to both the experimental and contrast groups. Subjects answered three questions on a five-point Likert scale. Questions and the average scores for each group are shown in Table 2.

Question 1-1 asked the subjective difficulty of summary writing, where 1 on the Likert scale indicated "very easy" and 5 "very difficult". The contrast group average was 2.50 (SD: 0.93) and the experimental group average was 2.38 (SD:0.74). Question 1-2 asked the exhaustiveness of the written summary from the subjects" own points of view, 1 indicating "not exhaustive at all" and 5 indicating "very exhaustive". The contrast group average was 4.13 (SD: 0.83) and the experimental group average was 4.25 (SD: 0.46). Question 1-3 asked the subjective effect of summary writing in gaining an overview of the topics learned in their investigation, 1 indicating "not effective at all" and 5 indicative "very effective". The contrast group average was 4.38 (SD: 0.52 ) and the experimental group average was 4.50 (SD: 0.53 ).

Table 2. Results of questionnaire \#1

\begin{tabular}{|c|c|c|c|}
\hline Question number & Question & $\begin{array}{l}\text { Contrast group } \\
\text { average }\end{array}$ & $\begin{array}{l}\text { Experimental group } \\
\text { average }\end{array}$ \\
\hline$\overline{Q 1-1}$ & How difficult was the summary writing? & 2.50 (SD: 0.93$)$ & 2.38 (SD:0.74) \\
\hline Q1-2 & $\begin{array}{l}\text { How much were you able to cover what } \\
\text { you investigated in the summary? }\end{array}$ & 4.13 (SD:0.83) & 4.25 (SD: 0.46) \\
\hline Q1-3 & $\begin{array}{l}\text { Did writing the summary help you } \\
\text { achieve an overview of what you } \\
\text { investigated? }\end{array}$ & 4.38 (SD:0.52) & 4.50 (SD: 0.53$)$ \\
\hline
\end{tabular}

\subsubsection{Questionnaire \#2}

Questionnaire \#2 was asked only to the experimental group and consisted of the following three sections: A) ease of using iLSB to create text structures with graphic organizers, B) effect of creating text structures with graphic organizers, and C) process of summary writing with graphic organizers. Questions were either answered by a five-point Likert scale or open-ended questions. The following tables will show average scores for the former. Open-ended questions are marked with "-" in the table.

Table 3 shows the results for questions concerning the use of iLSB (section A). For this section, 1 on the Likert scale indicated "very difficult", and 5 "very easy". First, the overall ease of using iLSB scored an average of 4.1 (SD: 0.83). From the open-ended descriptions answers to Q2A-2, it was observed that subjects perceived some difficulty when entering link annotations, viewing node annotations or the list of graphic organizers, and moving between graphic organizers to enter annotations. One subject commented that the GOs generated by iLSB was different from what the subject predicted, and that this led to some difficulty especially when adding annotations. The ease of entering node annotations averaged 4.1 (SD: 0.99) and 3.8 (SD: 1.58) respectively. For the visual ease of using the list of graphic organizers, the average was 4.1 (SD: 0.99). 
Table 3. Questionnaire results regarding the use of iLSB

\begin{tabular}{llll}
\hline Question number & Question & Average & SD \\
\hline Q2A-1 & $\begin{array}{l}\text { Was the iLSB easy to use for creating text structure with } \\
\text { graphic organizers? } \\
\text { (continued from previous question) What were the }\end{array}$ & 4.1 & 0.83 \\
Q2A-2 & & - \\
& difficulties? & 4.1 & 0.99 \\
Q2A-3 & Was it easy to enter node annotations? & 3.8 & 1.58 \\
Q2A-4 & Was it easy to enter link annotations? & 4.1 & 0.99 \\
Q2A-5 & Was the list of graphic organizers easy to grasp visually \\
& when writing the summary? & & \\
\hline
\end{tabular}

Table 4 shows the results to questions regarding the learning effect of GOs. Questions $2 \mathrm{~B}-1$ and $2 \mathrm{~B}-2$ asked whether GOs contributed to organizing information to determine relevant information for the summary. Results from Q2B-1 show that GOs on average scored 3.8 (SD: 1.04) on the effectiveness of organizing information from TOC on a Likert scale where 1 indicated "not helpful at all" and 5 "very helpful". Similarly, results from Q2B-2 showed the effectiveness of GOs in organizing the entire information on iLSB such as the learning scenario and TOC to be 4.1 (SD: 1.04). Q2B-3 aimed to measure how well the subjects understood the text structure represented by the GOs on a Likert scale with 1 indicating "could not understand at all" and 5 "understood very well". The average for this question was 4.0 (SD: 0.93). Finally, Q2B-4 was an open-ended question which aimed to identify factors of GOs to be improved in future research. Subjects raised the following points as difficulties in using GOs:

- inability to change GOs from those generated

- necessity of the GOs to be one of the five types

- not being used to GOs in general and therefore needing to check the type of text structure represented by each $\mathrm{GO}$

Table 4. Questionnaire results regarding the learning effect of GOs

\begin{tabular}{llll}
\hline Question number & Question & Average & SD \\
\hline Q2B-1 & Did GOs help you organize information in TOC? & 3.8 & 1.04 \\
Q2B-2 & $\begin{array}{l}\text { Did GOs help you organize information from your } \\
\text { investigation? }\end{array}$ & 4.1 & 1.04 \\
Q2B-3 & $\begin{array}{l}\text { How well did you understand text structures and other } \\
\text { information represented by GOs? }\end{array}$ & 4.0 & 0.93 \\
Q2B-4 & What were the factors of GOs that made it difficult to use? & - & - \\
\hline
\end{tabular}

Table 5 shows the results for questionnaires regarding the process of summary writing with GOs. Questions $2 \mathrm{C}-1$ and $2 \mathrm{C}-2$ asked how much subjects used the information in node and link annotations, respectively. The average scores on a Likert scale with 1 indicated "not useful at all" and 5 "very useful" were 4.6 (SD: 0.52) for the node annotations and 4.1 (SD: 0.99) for link annotations.

Q2C-3 asked the perceived effectiveness of the GOs in writing an exhaustive summary. Subjects answered on a Likert scale with 1 indicating "not effective at all" and 5 "very effective". The average score for this question was 3.9 (SD: 0.83). Q2C-4 asked the specific factors contributing the effectiveness, to which subjects listed the following:

- no need to take notes for writing

- visualization of the thought process

- visualization of the relationships and relevance between topics

- ease of ordering the topics in the summary

- ability to gain an overview of the topics

Q2C-5 asked the difficulty of writing text from the GOs to which subjects answered on a Likert scale with 1 indicating "very easy" and 5 "very difficult". The average score was 1.9 (SD: 0.64). Q2C-6 asked factors which made the above process difficult. Some subjects commented that as nodes in the GOs are represented with keywords, there was some difficulty in creating sentences from keywords and recalling what they learned from the investigation. Q2C-7 asked for comments regarding summary writing with GOs in general. It was observed that the ability to visualize, record, and organize information from the investigation as well as their own ideas was perceived to be an advantage of GOs, and that subjects overall felt GOs made summary writing easier. 
Table 5. Questionnaire results regarding the process of summary writing with GOs

\begin{tabular}{llll}
\hline Question number & Question & Average & SD \\
\hline Q2C-1 & $\begin{array}{l}\text { How useful were the node annotations for writing the } \\
\text { summary? }\end{array}$ & 4.6 & 0.52 \\
Q2C-2 & $\begin{array}{l}\text { How useful were the link annotations for writing the } \\
\text { summary? }\end{array}$ & 4.1 & 0.99 \\
Q2C-3 & $\begin{array}{l}\text { How effective were the GOs in writing an exhaustive } \\
\text { summary? }\end{array}$ & 3.9 & 0.83 \\
Q2C-4 & $\begin{array}{l}\text { What were the factors of GOs that made it easy to write } \\
\text { text? }\end{array}$ & - & - \\
Q2C-5 & $\begin{array}{l}\text { Was writing text from GOs difficult? } \\
\text { What were the factors of GOs that made it difficult to write } \\
\text { text? }\end{array}$ & - & 0.64 \\
Q2C-7 & $\begin{array}{l}\text { Please write any other comments on GOs and summary } \\
\text { writing. }\end{array}$ & - & - \\
\hline
\end{tabular}

\subsection{Discussion}

We will discuss the case study results with respect to the two aims of our method, which are 1) to support writing an exhaustive summary and 2) to decrease the cognitive load of the writing process. For the first aim, the experimental group was expected to create longer and more exhaustive summaries in less time compared to the contrast group using the GOs. However, results from the case study revealed no statistically significant differences. By observing individual data, this can be partly attributed to subjects in the contrast group already having high skills of organizing information and summarizing the information in text. For example, while the experimental group performed well on the summary's exhaustiveness, covering on average $96 \%$ of the q-keywords in TOC in their summary, the contrast group average also reached $91 \%$. By looking at the number of q-keywords in TOC which were not included in the summary, the experimental group subjects omitted 0 or only 1 keyword(s) except from one subject who omitted 2 keywords. For the contrast group 7 out of 8 subjects in the contrast group also omitted 0 or only 1 keyword(s), resulting in the two groups having similar results.

Results from questionnaire \#1 also support the hypothesis that the contrast group already had high writing and summarizing skills. From Q1-1, it can be observed that the perceived difficulty of summary writing was the same for both contrast and experimental group even though the contrast group had no support for the learning process. It can be said that some subjects in the contrast group did not require learning support for summary writing in WBIL and therefore were not targets of our research. As such, we conclude that the experimental group could write a summary in which the learners explained all or most of the q-keywords in TOC.

From the above evaluation, it can be concluded that using GOs to support summary writing was partially successful in achieving the two goals of the support set in our research. However, further research is required to evaluate the difference between the process where GOs are not used, especially those with a larger number of subjects and subjects targeted in our support.

Finally, reviewer assessments revealed that compared to the low-skill level subgroup of the contrast group, the experimental group's summary better reflected TOC. The utility of the application used for the learning support is evaluated from section A of questionnaire \#2, whose results indicate to iLSB was relatively easy to use and that the presentation of the information was clear.

\section{CONCLUSION}

Web-based investigative learning (WBIL) has been proposed as a method to support learners in effectively gathering information from the Web and building knowledge from the information. With the cognitive tool interactive Learning Scenario Builder (iLSB), learners can structure their processes according to the model designed in previous studies and use such functions as visualizing navigation paths and saving keywords from Web resources. This paper targets the process of summary writing in WBIL, which is an essential process for learners to share their learning outcomes to their peers or instructors. 
In our previous work, we have designed a method for helping learners build text structure with graphic organizers that allows learners to write a summary for their investigation in WBIL. Five types of graphic organizers which correspond to the five basic structures (description, sequence and order, cause and effect, compare and contrast, and problem and solution) were used to reorganize nodes included in TOC created during the knowledge construction process in WBIL. Our current work focused on the evaluation of this method. From the case study, it was suggested that text structure with graphic organizers were effective as a scaffold for writing a comprehensive summary with less effort. While differences between the contrast and experimental group were not found to be statistically significant, questionnaire results from the experimental group showed an overall positive attitude toward the method. Subjects observed that graphic organizers helped them by visualizing the order and relationships among information to be written in the summary. They also noted that writing the summary led to gaining an overview perspective of what they learned in their investigation. Along with the data obtained from analyzing the summaries, we have concluded that graphic organizers were able to decrease learners' cognitive load in writing a summary and making its contents exhaustive.

As the statistical insignificance for the results arose partly from the experimental design, where there was not enough control over the individual subjects' writing/organizing skills, further evaluation is necessary. In our future work, we plan to conduct a refined case study with more control over subjects' skills as well as a larger sample size.

\section{ACKNOWLEDGEMENT}

The work presented here is partially supported by JSPS KAKENHI Grant Number 17H01992 and $18 \mathrm{H} 01053$.

\section{REFERENCES}

Aramaki, M. et al, 2016. A Web-based Investigative Learning Support System (in Japanese), Proceedings of the 78th National Convention of IPSJ, Vol. 1, pp. 673-674.

Harada O. and Kashihara, A. 2020. A Graphic Organizer for Supporting Summary Writing in Web-based Investigative Learning, The 17th International Conference on Cognition and Exploratory Learning in Digital Age (CELDA 2020), Lisbon, Portugal (in press).

Julien H. and Barker, S. 2009. How high-school students find and evaluate scientific information: A basis for information literacy skills development. Library \& Information Science Research, Vol. 31, No. 1, pp. 12-17.

Kashihara, A. and Akiyama, N., 2016. Learning scenario creation for promoting investigative learning on the Web, The Journal of Information and Systems in Education, Vol. 15, No. 1, pp. 62-72.

Roehling, J. et al, 2017. Text structure strategies for improving expository reading comprehension. The Reading Teacher, Vol. 71, No. 1, pp.71-82.

Morishita, N. et al, 2020. Reconstructing Question tree in Web-based Investigative Learning. 44 ${ }^{\text {th }}$ Annual Conference of Japanese Society for Information and Systems in Education. in Japanese. Hamamatsu, Japan, pp. 169-176

Nishihara, Y. et al, 2006. Information Understanding Support by Using Informational complexity and Continuity (in Japanese). IEICE TRANSACTIONS on Information and Systems, Vol. 89, No. 9, pp. 1963-1975.

Zhang, K., \& Shasha, D. 1989. Simple fast algorithms for the editing distance between trees and related problems. SIAM journal on computing, Vol. 18, No. 6, pp.1245-1262. 